\title{
Meurtriers, jumeaux et devins : Trois variations sur le thème du double (Bassar, Togo)
}

Murderers, twins and diviners. Three variations on the theme of the double among the Bassar (Togo)

\section{Stéphan Dugast}

\section{(2) OpenEdition}

Journals

Édition électronique

URL : http://journals.openedition.org/span/1528

DOI : $10.4000 /$ span. 1528

ISSN : 2268-1558

Éditeur

École pratique des hautes études. Sciences humaines

Édition imprimée

Date de publication : 1 août 1996

Pagination : 175-209

ISSN : 0294-7080

Référence électronique

Stéphan Dugast, « Meurtriers, jumeaux et devins : Trois variations sur le thème du double (Bassar Togo) », Systèmes de pensée en Afrique noire [En ligne], 14 | 1996, mis en ligne le 17 avril 2014, consulté le 10 octobre 2020. URL : http://journals.openedition.org/span/1528; DOI : https://doi.org/10.4000/ span. 1528

Ce document a été généré automatiquement le 10 octobre 2020.

(c) École pratique des hautes études 


\title{
Meurtriers, jumeaux et devins : Trois variations sur le thème $\mathrm{du}$ double (Bassar, Togo) ${ }^{1}$
}

\author{
Murderers, twins and diviners. Three variations on the theme of the double \\ among the Bassar (Togo)
}

Stéphan Dugast

Lorsqu'ils abordent l'épineuse question des jumeaux et des procédures rituelles à mettre en œuvre pour rendre viable la cohabitation avec des êtres aussi hors du commun, les plus avisés des informateurs bassar évoquent le temps où, les ritualistes des jumeaux étant encore inexistants chez eux, l'affaire était mise entre les mains d'un autre spécialiste rituel, celui des meurtriers. D'emblée un lien explicite est ainsi établi entre le domaine des naissances hors normes et celui de l'homicide. Une telle présentation invite naturellement à s'interroger sur la nature de ce lien tout en suggérant d'appréhender la question du traitement rituel du meurtrier à la lumière des faits relatifs aux jumeaux

2 Autrefois donc, le dispositif rituel qui, de nos jours, permet d'intégrer à la communauté les jumeaux et tous les nouveau-nés qui leur sont assimilés était inconnu des Bassar. De tous les enfants ainsi marqués par les traits singuliers de leur naissance, les jumeaux étaient toutefois ceux qui plongeaient le plus leurs parents dans l'embarras: qu'une femme puisse mettre au monde deux enfants simultanément paraissait le comble de l'insolite. Pour cette raison, les nourrissons étaient confiés à un spécialiste des meurtriers, à charge pour lui de les enterrer dans une termitière en brousse. Seul un tel personnage pouvait se risquer à commettre un acte aussi grave qui l'exposait à la vengeance des petits êtres mis à mort ${ }^{2}$. Jabun, la puissance des meurtriers dont il est détenteur, celle-là même à laquelle doit avoir recours tout homicide aussitôt après le meurtre qu'il a commis s'il veut espérer échapper à la vengeance de l'esprit de sa victime, lui assurait l'indispensable protection ${ }^{3}$. Ce n'est que plus tard, lorsque les Bassar empruntèrent à leurs voisins kotokoli les rites et la médecine des jumeaux, qu'ils furent libérés de l'obligation de les mettre à mort. C'est en raison de ces 
anciennes pratiques, concluent les informateurs, que, dans la configuration actuelle, la médecine des meurtriers et celle des jumeaux sont associées.

De fait, l'analyse formelle des deux complexes rituels montre qu'ils relèvent du même ensemble, celui des puissances médicinales détenues à titre individuel par un spécialiste. Les procédures rituelles associées à ces différentes puissances présentent entre elles de nombreuses similitudes, notamment formelles, qui sont simultanément autant de caractéristiques qui les distinguent des autres ensembles rituels observables chez les Bassar. Tout invite donc à considérer séparément ce qui apparaît comme une classe autonome de rites (cf. Dugast 1992 : chap. VI). Pour autant, avec l'examen plus approfondi de certaines séquences, l'apparente homogénéité de cette classe se révèle n'être que relative: des différences sensibles apparaissent, créant en son sein une diversité notable. En pareille circonstance, la question se pose naturellement de savoir dans quelle mesure cette diversité est le reflet d'une hétérogénéité irréductible, susceptible par conséquent de malmener l'unité de l'ensemble, ou, au contraire, est le produit de distinctions significatives qui, alors, renforcent la cohérence de cet ensemble tout en en élargissant le champ. De la réponse à cette question dépendra l'interprétation à donner aux spécificités que présente, au sein de cette classe de rites, le traitement du meurtrier.

Pour nous aider à y répondre, nous joindrons aux deux complexes déjà mentionnés (celui du meurtre et celui des naissances singulières) un autre relevant du même ensemble : le domaine de l'initiation à la divination. La prise en compte de ce troisième exemple nous permettra, par comparaison avec les deux précédents, de rendre discernables des opérations rituelles qui autrement paraissent confusément entremêlées. Celles dont relève plus spécifiquement la cure du meurtrier pourront ainsi être mieux identifiées.

\section{Le meurtre et ses conséquences}

5 Tout acte d'homicide expose immédiatement le meurtrier à la vindicte de l'esprit de sa victime. Sa seule chance d'en réchapper est de s'adresser sans attendre à un spécialiste rituel, détenteur de la puissance appropriée (jabun), auprès duquel il accomplira l'indispensable cure. C'est au cours de cette cure que seront effectuées les principales opérations rituelles destinées à assurer sa protection.

Le spécialiste sollicité, jabundaan (litt. "le détenteur de jabun »; le suffixe -daan désigne la qualité de détenteur), est, chez les Bassar, un ritualiste de premier plan. Si les cas de meurtre constituent son principal secteur de compétence, ce personnage intervient également dans le traitement d'une vaste catégorie de mauvais morts. Son rôle dans ce domaine est d'autant plus important que, à la différence de la plupart des autres sociétés ouest-africaines, les Bassar reconnaissent à de tels morts la possibilité d'accéder au statut d'ancêtre dès lors que sont accomplis certains rites de « rattrapage ». Ces rites relèvent, pour les uns, de la compétence de ce personnage et, pour les autres, de celle d'un autre spécialiste, un maittre de la foudre. Dans tous ces cas, il s'agit de transformer le mauvais mort en mort ordinaire, opération à partir de laquelle pourra, plus tard, être entrepris le procès d'ancestralisation dans son cours normal. Tandis que les maîtres de la foudre ont une compétence qui se limite aux foudroyés, les détenteurs de jabun interviennent pour plusieurs types de mauvais morts. Ceux-ci se divisent principalement en deux grandes catégories: d'une part les 
"brûlés », que sont les victimes d'homicide ou de morsures de serpent, mais aussi les lépreux, et d'autre part les "enflés ", catégorie qui regroupe tous ceux dont le corps, au moment du décès, est affligé d'un enflement quelconque (les femmes enceintes en constituent bien évidemment un exemple typique) ${ }^{4}$.

7 Ces deux catégories sont directement liées au meurtre car, si la victime d'un homicide est donc un "brûlé ", de son côté, le meurtrier est menacé de troubles divers dont le plus manifeste est un enflement considérable de son corps qui s'emplit de titaykpiilool, le "poison des ancêtres" (utajkpiil, "ancêtre», tilool, "poison»). Au feu auquel a succombé la victime s'oppose donc le liquide qui emplit le corps du meurtrier et met sa vie en péril. En outre, ce symptôme fait de ce dernier un «enflé » qui, en cas d'issue fatale (inévitable dans le cas où la protection de jabun ne serait pas sollicitée ou se révélerait impuissante), sera un mauvais mort dont le "rattrapage » incombera à un jabundaan, au même titre donc que celui de sa victime.

8 Ces symptômes qui affectent le corps de l'homicide sont le signe que la victime, ou plus précisément son principe vital, le kinan, est parvenu à s'introduire dans le corps de son meurtrier : «U kJo-u », « il (la victime) est entré en lui (l'homicide) ». Dès les premiers instants qui suivent le meurtre, le kinan de la victime tente en effet de s'introduire à l'intérieur de son tueur. C'est à cette tentative que la cure doit faire échec. Si cette dernière n'est pas accomplie à temps, le meurtrier sera hanté en permanence par l'image de sa victime, il la verra cherchant sans cesse à l'attaquer. Dans des cas moins extrêmes, c'est surtout lors des rêves que la victime se manifeste. Parfois, ces manifestations sont le signe que le jabun auquel le meurtrier a eu recours est insuffisamment puissant pour le cas à traiter. Le patient devra alors se tourner vers un autre détenteur de jabun dont la puissance sera plus efficace et lui assurera une meilleure protection. S'il ne le fait pas, il sera sujet à des malaises croissants qui le feront dormir sans interruption, cependant que son corps se mettra à blanchir, ses joues à gonfler ; et, si toujours rien n'est fait, il finira par mourir avec le corps enflé.

9 Là s'arrête, chez les Bassar, la liste des principales caractéristiques de l'homicide. À la différence de ce que l'on observe dans de nombreuses sociétés, y compris chez les voisins immédiats des Bassar, le sang du meurtre est dit n'avoir aucune incidence sur le sort du meurtrier. Cette question du sang est du reste l'une des rares qui distingue les représentations bassar de celles des Konkomba en ce qui concerne le meurtre. Les Konkomba, voisins septentrionaux des Bassar, et très proches d'eux culturellement, conçoivent en effet le sang de la victime comme un élément susceptible d'aggraver l'état du tueur s'il vient au contact de ce dernier : le kinan de la victime se colle au corps du meurtrier comme le sang qui l'a aspergé (Froelich 1964 : 205-206). Cette mention d'un kinan qui parvient à établir un contact intime avec le corps de l'homicide en adhérant à sa peau est, tout autant que la référence au sang, étrangère aux conceptions bassar : selon ces dernières, on l'a vu, c'est avant tout par intromission que le kinan de la victime cherche à faire corps avec son meurtrier afin de lui nuire. Une troisième différence doit être signalée dans le prolongement de ces deux premières: la conséquence de cet épanchement du sang de la victime sur la peau du meurtrier est, chez les Konkomba, d'aggraver la lèpre dont il sera de toute façon frappé à la suite de son acte. C'est en effet la lèpre, et non un enflement comme chez les Bassar, qui constitue dans cette population la principale pathologie qui affecte l'homicide.

Dans le sillage de la question du sang, on voit donc apparaître deux autres spécificités des représentations konkomba : les trois éléments forment un ensemble solidaire dans 
la mesure où tous trois sont livrés simultanément, à propos du même événement que constitue le contact entre le sang de la victime et le corps du meurtrier. On ne peut pour cette raison envisager la question de la référence au sang sans y intégrer les deux autres spécificités qui l'accompagnent. Or, celles-ci sont de toute évidence le produit de transformations significatives à partir de la configuration bassar (ou inversement) : substituer, dans le premier cas, la lèpre aux enflements, c'est en effet simplement permuter deux termes qui, on l'a vu, sont pensés dans le symbolisme bassar de jabun comme relevant de deux catégories opposées et complémentaires, et comme telles mises en relation; de même, substituer, dans le second cas, une agression externe par adhésion sur une surface corporelle à une agression interne par intromission est une opération qui ne fait que mobiliser des représentations identiques à celles à l'œuvre dans le domaine du traitement du meurtrier tel que le mettent en œuvre les Bassar. Nous verrons plus loin en effet que la cure bassar articule un traitement externe d'une enveloppe corporelle à un traitement interne au moyen d'une absorption alimentaire. La même articulation logique semble donc rendre compte du rapport entre les représentations des Bassar et celles des Konkomba quant aux moyens par lesquels le kinan de la victime parvient à s'immiscer dans l'intimité de son meurtrier.

11 Si donc la question du sang ne peut être envisagée que dans son association étroite avec les traits que lui attachent les Konkomba, on doit admettre qu'elle ne constitue que l'un des éléments d'un champ de transformations qui permet de passer logiquement des représentations konkomba à celles des Bassar. Que le sang ne soit pas explicitement reconnu comme pertinent chez ces derniers n'exclut par conséquent pas qu'il le soit virtuellement. La série retenue par les Bassar, associant les enflements, l'intromission au sein du corps, et enfin les aliments, en l'absence de toute référence au sang, n'est en définitive que l'un des termes d'une alternative qui comprend également la série opposée associant, comme forme dominante d'agression du kinan de la victime à rencontre du meurtrier, la lèpre, l'adhérence à une surface corporelle, et enfin le sang.

Un autre trait fréquemment observé en matière de traitement rituel des meurtriers se signale également par son absence chez les Bassar : la croyance selon laquelle l'état du meurtrier est contagieux, risquant de contaminer son entourage s'il n'est pas isolé jusqu'à ce que son état soit résorbé. Là encore, on trouve une expression de ce trait chez les Konkomba voisins, où elle s'articule d'ailleurs étroitement avec la question du sang. Après un homicide, les vieux de la maison du meurtrier sortent sur la cour extérieure où celui-ci est contraint de rester. Ils « mettent un peu de médicament dans l'eau, tournent avec la main gauche, boivent et font boire le garçon, sinon leur ventre gonflerait» (Froelich 1954 : 205). En d'autres termes, la contamination à laquelle le meurtrier expose ses proches, menaçant de les rendre sujets à d'inquiétants enflements, ne peut être combattue que par l'absorption d'un médicament. C'est là une situation qui rappelle, par transformation de ses principaux termes, celle que décrivent les Konkomba à propos du meurtre lui-même : le sang de la victime provoque (ou en tout cas aggrave) la lèpre chez le meurtrier si d'aventure il se répand sur la surface de son corps. Entre les deux situations, les relations font en effet intervenir une nouvelle fois les deux oppositions déjà identifiées: celle entre les catégories de la lèpre (affectant le meurtrier) et des maladies à enflement (menaçant son entourage) d'une part, et celle entre une application externe sur une enveloppe corporelle et un traitement interne par absorption alimentaire d'une substance médicamenteuse d'autre part. Les effets du contact avec le sang empruntent les formes définies par la première série (lèpre par contact dermique), tandis que les effets de la contamination 
sont caractérisés par les termes de la seconde série (gonflements et absorption alimentaire). Le sang auquel est exposé le meurtrier et la contamination dont est menacé son entourage sont donc deux éléments liés par des rapports complexes, qui font intervenir deux séries contrastées de termes associés.

Ces deux séries correspondent à une opposition générale qui structure une grande partie du champ symbolique du meurtre que partagent les deux populations, bassar et konkomba. La double absence, chez les Bassar, de la référence au sang et du discours sur la contamination peut ainsi être envisagée comme l'effet d'une même transformation logique. En déniant toute pertinence au sang de la victime, les Bassar écartent simultanément les traits qui lui sont associés chez les Konkomba, à savoir la lèpre et le contact externe entre le kinan et le meurtrier. Pour caractériser les maux qui affectent ce dernier, ne sont alors plus disponibles que les termes de la seconde série (enflements et intromission), lesquels ne peuvent du coup plus être sollicités pour caractériser une contamination qui n'a plus sa place.

Le sang du meurtre se trouvant dépourvu, chez les Bassar, de toute pertinence, on ne s'étonnera pas que la distinction entre meurtre sanglant et autres formes d'homicide ne soit pas pertinente : tous requièrent, au même titre, l'accomplissement de la cure de jabun. Le rite bassar ne tient pas compte non plus d'autres distinctions souvent à l'œuvre: ainsi celle entre endo-meurtre et exo-meurtre, tout comme celle entre meurtre intentionnel et meurtre par accident.

La relation initiale entre le meurtrier et sa victime, ou entre leurs lignages respectifs, n'a en effet aucune incidence sur le risque qu'encourt le premier ni sur les modalités de la cure à laquelle il doit s'astreindre. Cette relation fait néanmoins, dès lors que le meurtre a été commis, l'objet de certains interdits. En premier lieu, le meurtrier doit désormais s'abstenir de manger toute nourriture ou de boire toute boisson provenant de l'une des cours du lignage de sa victime. En second lieu, les mariages entre les deux lignages deviennent impossibles. La transgression de ces interdits entraînerait la mort des contrevenants, victimes de la sanction des ancêtres. Enfin, les femmes mariées de chacun des deux lignages qui sont enceintes au moment du meurtre ne doivent plus rendre visite à une famille de l'autre lignage, et ce pour quelque motif que ce soit. Le feraient-elles, qu'elles risqueraient de donner naissance à un enfant dépourvu de sexe. On dit que l'enfant perd son sexe, "ça a coupé l'enfant» (an gii kibiki), qu'il ait été destiné à être une fille ou un garçon : "L'enfant qui va naître, s'il était un garçon, il perd son sexe, il n'est plus garçon, et il n'est pas non plus une fille. S'il était une fille, elle perd son sexe, elle n'est plus une fille, et elle n'est pas non plus un garçon ».

La distinction entre meurtre d'un proche et meurtre d'un étranger, éventuellement ennemi, ainsi que celle entre meurtre accidentel et meurtre intentionnel sont d'ailleurs si peu pertinentes dans le rite de jabun que ce dernier peut être mis en œuvre dans des circonstances où toute notion d'agression est totalement absente. Jabun peut en effet être sollicité en appoint des rites de deuil lorsque ceux-ci intéressent une personne ayant entretenu avec le défunt des relations particulièrement intimes. C'est le cas d'un enfant en bas-âge qui serait resté très attaché à sa mère dont l'esprit viendrait le hanter, causant pleurs et cauchemars. Ou encore, pour un jeune couple dont l'un des membres viendrait à disparaître prématurément, on soumet le survivant au rite de jabun afin de mieux détacher de lui l'esprit de son conjoint défunt. Dans les deux cas, jabun vient en complément des rites de deuil car, nous le verrons, il est parmi les plus puissants rites de séparation dont les Bassar disposent. Surtout, il est particulièrement 
adapté à toutes ces situations où un esprit à écarter entretient avec l'intéressé une relation très intime. De ce rapprochement, on peut déduire que le meurtre instaure, entre l'homicide et sa victime, une relation de soudaine intimité qui appelle une vigoureuse séparation.

\section{La puissance de jabun}

17 Jabun fait partie de cet ensemble de complexes rituels qui, chez les Bassar, sont à la fois considérés comme des puissances (mpon, litt. "force», de $p \supset$, «être fort») et des médecines (nnyכkJ, de kunyokJ, « bouche »; ce terme désigne donc « ce qui est destiné à être avalé»). Cette double appellation s'applique indistinctement à toutes les puissances médicinales, qu'elles soient individuelles (comme pour les trois complexes qui sont présentés ici) ou collectives (lesquelles relèvent d'une classe voisine mais distincte, cf. Dugast 1992 : chap VII). Elle ne s'applique en revanche à aucun autre type de puissance surnaturelle telles que celles que constituent les ancêtres ou encore ces entités inscrites dans le paysage que les Bassar nomment awaa (cf. Dugast 1992: chap. VIII). Comme tous les complexes rituels de sa catégorie, celui de jabun fait intervenir un certain nombre d'objets spécifiques dont les noms peuvent servir accessoirement à le désigner. Au terme de jabun sont donc souvent préférés ceux de $\eta f a n$, «foyer » (en référence au foyer spécifique utilisé pour la cuisson de la nourriture rituelle, foyer dont la particularité est d'être composé de trois pierres en latérite incrustées dans le sol de la cour du détenteur) et de yyin, "corne" (par allusion à la corne d'antilope qui contient la poudre médicamenteuse associée à la puissance de jabun). En référence à ces différents termes, le détenteur de cette puissance pourra indifféremment être appelé jabundaan, yfandaan, ou yyindaan.

18 On compte dans chaque village ou agglomération plusieurs détenteurs de jabun, chacun ayant la possession d'une puissance qui lui est propre. La distribution des puissances de jabun au sein d'une localité ne répond à d'autres critères que ceux qui résultent de l'histoire particulière de chacun de leurs détenteurs : le statut de jabundaan est acquis de manière strictement individuelle, l'appartenance à une unité clanique ou lignagère, décisive en d'autres circonstances, n'est ici d'aucune pertinence. On peut donc compter plusieurs puissances de jabun au sein d'un même lignage et n'en trouver aucune dans plusieurs autres lignages voisins, sans que de telles disparités aient le moindre effet sur le statut des unités sociales concernées ${ }^{5}$. Chaque puissance particulière possède en propre plusieurs traits distinctifs, repérables notamment dans certaines variantes secondaires des procédures rituelles mises en œuvre. Mais le plus notable de ces traits distinctifs est vraisemblablement le nom propre donné à la puissance elle-même, nom qui doit être proféré à un moment crucial de la cure. Nous y reviendrons.

19 Respectés en raison des nombreux services qu'ils rendent, les détenteurs de jabun sont simultanément craints du fait de leur maîtrise d'une puissance qui, dans certaines occasions, peut se montrer redoutable. Si jabun a en effet pour vocation première d'assurer une protection à certaines personnes menacées, elle est tout aussi capable de s'attaquer à ceux qui entretiendraient avec elle un rapport non motivé. Dans ces circonstances, l'agressivité que libère jabun présente des traits comparables à ceux qui caractérisent le kinan brutalement libéré d'une victime d'homicide. Ainsi, au cas où une querelle oppose un jabundaan - ou même un homme qui a simplement accompli la cure de jabun - à un homme «ordinaire ", certains gestes sont à éviter. Par exemple, si au 
cours de cette querelle le premier défie le second en lui disant : " ose taper dans ma main si, comme moi, tu es un homme!» (ou encore, tenant dans la main gauche un objet quelconque, il met son adversaire au défi de le lui prendre) et si, enfin, ce dernier relève le défi, il sera victime des maux de jabun et, à terme, si rien n'est fait pour le sauver, il mourra enflé. Comme dans tous les contextes dominés par jabun, le moindre des remèdes consiste à faire lécher à l'imprudent la poudre médicinale. De telles situations font ainsi apparaître, par le type de symptômes aussi bien que par le type de traitement, une singulière ressemblance entre la force qui émane de jabun et celle que véhicule le kinan d'une victime de meurtre.

De fait, jabun est d'abord une puissance agressive : elle ne protège que dans la mesure où elle s'attaque à une autre puissance, le kinan de la victime, pour la neutraliser. Tout est affaire de redoutables forces qui s'affrontent et entre lesquelles il faut trouver un équilibre. Les conditions d'acquisition d'une puissance de jabun sont évocatrices à ce propos. Ne devient pas jabundaan qui veut. Dans la relation qui lie un tel personnage à sa puissance, le meurtre est un élément essentiel. Si d'aventure un homme se risquait à faire l'acquisition d'une puissance de jabun sans avoir lui-même commis de meurtre, il s'exposerait à une mort certaine: sa puissance se mettrait en quête du kinan de sa victime afin de le combattre et, ne le trouvant pas, se retournerait finalement contre l'acquéreur illégitime. La mort qui frappe ce dernier présente alors les mêmes caractéristiques que celle qui est réservée à un meurtrier qui aurait omis de se mettre sous la protection d'une puissance de jabun : il succombera avec le corps enflé.

21 A l'inverse, de nombreux cas d'acquisition d'une puissance de jabun sont motivés par une cure qui, à la suite d'un meurtre, se montre impuissante à repousser durablement les assauts du kinan de la victime. C'est cette fois la force de ce kinan qui supplante celle de la puissance, et l'une des solutions, pour l'homicide, est de faire lui-même l'acquisition d'un jabun: avec l'instauration d'une relation aussi intime avec la puissance se renforce la protection assurée par cette dernière, ce qui a pour effet de restaurer l'équilibre des forces antagonistes.

Par conséquent, faire l'acquisition d'une puissance de jabun pour quelqu'un qui, faute de meurtre, n'a pas déclenché de kinan hostile, tout comme, à l'inverse, se contenter d'une simple cure de jabun alors que le kinan libéré se révèle particulièrement agressif, sont deux attitudes opposées qui conduisent l'intéressé à la même issue fatale. C'est que, dans cette relation d'affrontement, jabun du meurtrier et kinan de la victime apparaissent comme formant couple. L'un appelle l'autre, et réciproquement : de même que le meurtre impose la cure de jabun, l'acquisition de jabun requiert que l'intéressé ait commis un meurtre. Que l'un des termes soit présent en l'absence de son complémentaire, et le personnage responsable de ce déséquilibre se voit frappé de mort, pire, d'une mauvaise mort. Le fait que cette mort s'accompagne dans les deux cas des mêmes caractéristiques (enflement du cadavre du contrevenant) montre que ces deux puissances qui s'affrontent sont en outre de même nature. Un examen des opérations rituelles accomplies au cours de la cure permettra de mieux cerner sur quoi repose cette parenté.

Si l'acquisition d'une puissance de jabun par un homicide est généralement motivée par l'échec d'une première cure, un tel échec, on l'a vu, n'a pas toujours des conséquences aussi extrêmes. Les différentes puissances de jabun n'ayant pas toutes la même force, il suffit parfois de s'adresser au détenteur d'un jabun plus efficace. Pour reprendre les 
termes des Bassar, il faut trouver la corne (yyin) qui sera plus forte que la menace, celle qui sera en mesure de la détourner.

Afin d'agir plus efficacement contre le kinan de la victime, il est recommandé de connaître le nom propre du défunt. Si tel est le cas, dès que le meurtrier arrive chez le jabundaan, celui-ci décroche sa corne yyin, retire le bouchon qui la ferme pour en extraire la poudre médicinale qu'elle contient, et la donne à lécher au meurtrier. Après quoi, le jabundaan demande au meurtrier de prononcer trois ou quatre fois le nom de sa victime (trois fois s'il s'agit d'un homme, quatre s'il s'agit d'une femme ${ }^{6}$ ) en se plaçant face à la corne. Aussitôt après, le jabundaan referme la corne avec le bouchon : on a ainsi « attrapé la victime pour la mettre dans la corne ». À cette scène s'oppose celle qui se tiendra plus tard, au moment central de la cure, lorsque le meurtrier devra absorber la préparation médicinale appelée à le protéger : avant chaque bouchée, il lui faudra prononcer le nom propre de la puissance de jabun dont il a sollicité la protection. Les deux usages de ces noms propres ont des finalités contraires mais complémentaires : le nom de la victime est énoncé pour détacher du meurtrier ce kinan trop présent, celui de la puissance de jabun l'est pour instaurer, entre cette puissance et le meurtrier, une intimité proche de la consubstantialité.

Cette obligation de proférer le nom de la puissance au moment de l'ingestion du repas médicinal souligne simultanément la part prépondérante de l'acte du manger dans la cure. Certes, cela n'est guère étonnant s'agissant d'un rite « médicinal », où l'accent est naturellement mis sur l'absorption d'une certaine substance, mais ici le trait est encore plus appuyé. Par rapport à d'autres rites dont la part médicinale est tout aussi affirmée, celui de jabun contient en effet une multitude de précautions supplémentaires autour de toutes les opérations qui vont de la préparation culinaire à l'ingestion des nourritures préparées. Ainsi, le foyer lui-même, dont le nom peut servir, on l'a vu, à désigner le rite dans son ensemble, est un foyer spécifique, dont l'usage est exclusivement réservé à l'accomplissement de la cure. Cette exclusivité le signale comme recelant une partie de la puissance de jabun. C'est donc la cuisson même des nourritures qui se révèle une opération extrêmement délicate pour laquelle on ne saurait prendre assez de précautions. Si le foyer est à cet égard l'élément qui concentre la plus grande part de cette puissance émanant de jabun, il n'épuise pas à lui seul cette qualité. La marmite, elle aussi, est un ustensile spécifique, utilisé uniquement à l'occasion des rites de jabun. On l'appelle d'ailleurs « la marmite de jabun » (jabugkumbo ki). Le dépôt de ce récipient sur le foyer requiert en outre un petit cérémonial: le meurtrier et le détenteur de jabun le saisissent tous deux de la main gauche, effectuent ainsi trois tours au-dessus de l'endroit où a été immolé l'animal (là où sont entassés les objets de la puissance), puis viennent se placer des deux côtés du foyer où, de nouveau, ils font tourner trois fois la marmite au-dessus du feu.

Pour la cuisson de la viande, la sauce doit être remuée avec un os particulier, retiré du sac de jabun. Il s'agit en principe d'un os provenant du bras gauche d'un être humain? Mais, à défaut d'avoir pu s'en procurer un, opération dont on peut concevoir la difficulté, il est permis de se contenter d'un os de la patte avant gauche d'un lion, d'un léopard ou d'une hyène ${ }^{8}$. N'importe lequel des participants peut remuer la sauce avec cet « ustensile », mais il est impératif qu'il fasse alors usage de sa main gauche, et qu'il pousse le cri de guerre: «SamboJ!». Ensuite, pour retirer la marmite du foyer, de nouvelles précautions sont prises, combinées avec l'extinction du feu : le meurtrier et le jabundaan prennent dans la bouche de l'eau qui a été puisée le matin même au 


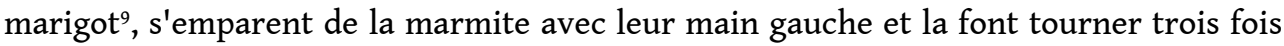
au-dessus du foyer, projettent sur le feu l'eau qu'ils avaient dans la bouche et déposent la marmite sur le côté. C'est à ce moment que prend place la séquence déjà évoquée où le meurtrier doit manger la nourriture rituelle en proférant le nom de la puissance. Il le fait dans une calebasse, elle aussi spécifiquement attachée au rite de jabun (on l'appelle jabunyuku, la «calebasse de jabun»), qui est posée sur le foyer, trait qui souligne une nouvelle fois la place centrale de cet élément et son rôle de protection autour des substances destinées à être absorbées par le patient.

Cette présentation sommaire des caractéristiques de la puissance de jabun fait donc apparaître que la cure du meurtrier accorde un rôle majeur au registre alimentaire. Ce constat nous amène à aborder l'aspect médicinal de la puissance d'autant que, comme toute médecine, celle de jabun est, pour une part essentielle, destinée à être absorbée par le patient. C'est d'ailleurs principalement au cours du repas dont il a été question que cette absorption se fera. Mais, chez les Bassar, une médecine intègre également d'autres composantes, et c'est cette configuration d'ensemble qu'il nous faut à présent examiner.

\section{La médecine de jabun}

Dans le déroulement du rite, le procès sacrificiel et le procès de préparation des denrées médicinales sont étroitement imbriqués. Le rite commence par le dépôt des objets de jabun sur le sol de la cour auxquels sont joints les végétaux récoltés pour la circonstance. Végétaux et objets rituels forment alors un amas d'éléments à peine différenciés qui seront traités de façon homogène durant les premières phases du rite. Tous recevront la libation préalable de farine de mil diluée, l'animal à immoler sera posé sur le tas qu'ils forment et c'est là qu'il sera égorgé, son sang devant couler sur chacun des éléments en présence ${ }^{10}$.

Comme dans la plupart des rites comprenant l'immolation d'un animal, la préparation et la cuisson de la viande introduisent une rupture dans le déroulement de la cérémonie. Elles séparent les deux principales phases d'offrandes, la première centrée sur le sang frais et, le cas échéant, les plumes ou poils de l'animal, la seconde sur la viande cuite, parfois accompagnée d'une sauce, obtenue au cours de la même cuisson. Dans le cas présent, cette rupture est mise à profit pour accomplir deux opérations : parallèlement à la préparation de la viande, on s'affaire autour des végétaux pour les transformer en produits médicinaux.

Ces végétaux sont composés de feuilles et de racines prélevées sur une série d'arbres ou d'arbustes soigneusement sélectionnés. Les racines sont épluchées de sorte que sont finalement distingués trois éléments : les feuilles, les bois des racines et leurs écorces. Chacun de ces éléments a sa destination propre.

Les bois des racines sont découpés en petits morceaux qui sont carbonisés au feu. Cette carbonisation est le principal traitement distinctif qui leur est réservé. Le charbon obtenu est pilé, puis écrasé sur une meule dormante. On y ajoute des graines de sésame, elles aussi moulues. Mais surtout, on y joint un ingrédient fondamental : une portion de foie humain préalablement séché par le jabundaan et pulvérisé pour l'occasion ${ }^{11}$. La poudre obtenue par l'assemblage de ces différents éléments sera versée dans la sauce de la viande en train de cuire, mais une partie sera mise en réserve pour les cas d'urgence, ainsi que pour un autre usage (le tracé de croix pour ponctuer un parcours, 
cf. infra). Deux des objets de jabun en constitueront les récipients : les gourdes et la corne.

Les écorces des racines sont quant à elles pilées sur une pierre en même temps qu'une autre portion de foie humain ${ }^{12}$. L'ensemble est ensuite écrasé sur une meule et le produit de cette mouture est tassé en forme de petite boule, laquelle sera conservée dans le sac de jabun. Elle pourra, dans certains cas, être donnée à lécher comme traitement d'urgence.

Les feuilles, enfin, sont déposées dans une large cuvette en terre cuite remplie d'eau où elles sont laissées en macération. À la fin du rite, on plante le couteau de jabun dans le tas de feuilles afin d'éviter que le kinan de la victime ne vienne introduire quelque chose dans l'eau. Cette macération sera utilisée pour un traitement complémentaire par le meurtrier qui devra se doucher avec durant trois ou quatre jours, selon le sexe de sa victime. À l'issue de cette période, la cuvette sera vidée à un carrefour.

Ces trois produits élaborés à partir de trois ensembles végétaux distincts ont des usages variés. Les deux plus importants sont les premiers et les derniers, qui se complètent : ils sont à la base d'une cure qui combine un traitement interne (par absorption de la poudre, ingérée en même temps que la nourriture "sacrificielle ») avec un traitement externe (par aspersions régulières de l'eau dans laquelle baignent les feuilles de jabun). Ce double traitement fait intervenir des éléments végétaux contrastés, feuilles d'une part, racines d'autre part, les unes plongées dans l'eau, les autres calcinées par le feu.

L'articulation entre les traitements interne et externe de la cure renvoie aux représentations relatives au meurtre et à ses conséquences. On se souvient qu'à une agression initiale et brutale qui s'assimile à une « brûlure externe » (le meurtre) répond une agression ultérieure plus insidieuse que ses caractéristiques (enflements, notamment par hydropisie) font apparaître comme une «inondation interne ». Pour neutraliser cette seconde agression, la parade consiste à suivre un traitement qui combine l'absorption d'un médicament "brûlé » (la poudre de racines carbonisées), donc quelque chose qui est de l'ordre d'une «brûlure interne ", avec l'aspersion d'un autre médicament liquide (la macération), donc quelque chose qui relève d'une « inondation externe ». À cet ensemble d'associations semble se rattacher le soin que prennent les acteurs du rite à asperger d'eau le feu de cuisson au terme de la préparation culinaire (cf. supra).

Si l'on examine enfin le cas des écorces de racines, on s'aperçoit qu'elles font figure de terme médiateur. Par leur composition d'abord: elles sont métonymiquement des racines, puisqu'elles en sont extraites, et, métaphoriquement, elles sont des " feuilles " puisqu'elles sont des enveloppes (celles des racines); or, dans la cure, les feuilles ont précisément pour objet de renforcer une enveloppe, celle du corps du tueur. C'est ensuite par leur mode de préparation qu'elles se révèlent comme intermédiaires : à l'instar des racines, elles sont pilées et moulues, mais elles ne sont pas carbonisées, de sorte que, comme les feuilles, elles conservent leur qualité de végétaux frais. C'est sans doute parce qu'elles rassemblent ces diverses propriétés que la boule qu'elles constituent est conçue comme fournissant à elle seule un traitement complet dans les cas les plus bénins : par exemple pour quelqu'un qui aurait rencontré un cadavre ou qui aurait été témoin d'un meurtre, ou encore dans certains cas de cauchemars répétés, il suffit de faire lécher la boule de jabun pour dissiper le mal ${ }^{13}$. 


\section{Une attention particulière portée au registre alimentaire} certaine cohérence d'ensemble, n'exclut pas la prépondérance de l'un d'entre eux, l'aspect alimentaire. Les Bassar sont unanimes à ce sujet: le principal stratagème mis en œuvre par la victime pour se venger consiste à profiter de la vulnérabilité du meurtrier à chacun des moments où il s'alimente. C'est en s'introduisant dans les aliments qu'il absorbe que le kinan de la victime espère pénétrer dans son corps. La première des précautions consiste donc à éloigner ce kinan de toutes les substances que le meurtrier est appelé à consommer. C'est l'objet de tous les interdits alimentaires qui lui sont imposés. Ainsi, il lui est vivement recommandé de ne manger aucun aliment après le coucher du soleil, moment à partir duquel le kinan de la victime se fait plus agressif. De même, les restes de plats lui sont interdits. On prétend en effet que, lorsque le soir tombe, le double de l'homme ou de l'animal abattu vient dans la demeure de son tueur et mange les restes de plats consommés dans la journée. Manger ces mêmes restes reviendrait à les partager avec la victime, et une telle commensalité, que l'on cherche précisément à éviter, pourrait être fatale. Le miel, la viande de rat ou de souris ne doivent pas non plus être consommés par l'homicide, pour les mêmes raisons : mais cette fois, on précise que ce sont des nourritures particulièrement appréciées par l'hyène, l'un des animaux dont la mise à mort est assimilée à celle d'un homme. La liste comporte en outre les animaux de toute espèce qui auraient été trouvés morts et ramassés par quelqu'un: cette fois, la référence est plus large et concerne tous les charognards ${ }^{14}$. Enfin, il est une précaution que ne saurait négliger à aucun moment le meurtrier : celle qui consiste à éviter qu'une mouche, ou tout autre insecte, ne vienne au contact d'un breuvage ou d'une nourriture qu'il s'apprête à ingérer; si cela se produisait, il devrait immédiatement cesser de boire ou de manger, car l'insecte pourrait n'être autre que la victime ainsi métamorphosée en vue précisément de s'introduire en lui à son insu.

Mais la plus efficace des précautions dans ce registre alimentaire est celle que dispense la cure de jabun elle-même, avec principalement la consommation du repas rituel au cours duquel s'instaure une forme d'intimité avec la puissance (intimité soulignée par de nombreux traits du rite dont l'obligation de proférer son nom propre avant chaque bouchée n'est pas le moindre). Cette intimité garantit au meurtrier la présence ultérieure de cette puissance à ses côtés : à chacun de ses prochains repas, elle sera ainsi en mesure de renvoyer le kinan de la victime, toujours à l'affût.

\section{Jabun, une puissance qui intègre la force d'un kinan}

Jabun et kinan, avons-nous dit, forment un couple de termes comparables mais antithétiques. Du seul fait de cette opposition, tout rapprochement vers jabun assure simultanément, pour une part essentielle, la mise à distance de son antagoniste, le kinan de la victime. C'est précisément ce qui s'opère dans les principales phases de la cure. Jabun est d'autant plus efficace dans sa neutralisation du kinan de la victime qu'elle participe de la même nature. Cette parenté s'est révélée notamment par le fait que, puissance en principe vouée à la protection, jabun se mue, en l'absence de menace extérieure, en une puissance qui tue, et selon des modalités identiques à celles qui 
suivent tout acte d'homicide. D'où provient cette parenté ? Plusieurs éléments entrevus lors de la description de la cure éclairent cette question. L'ingrédient de base de la poudre médicinale de jabun est une portion de foie humain. Or, pour les Bassar, le foie est le siège du kinan de chaque personne. Par la participation de cette substance, c'est donc la force d'un kinan humain qui est introduite dans la puissance-médecine de jabun. La même logique semble présider à l'obligation de mélanger la sauce à l'aide de l'os d'un bras humain. On se souvient d'ailleurs que, concernant l'origine de ces deux éléments, les informateurs ne sont pas tous unanimes. Certains vont jusqu'à affirmer que ces portions de cadavre humain doivent provenir d'un homme tué par flèche : le kinan que l'on cherche à insérer dans la puissance-médecine de jabun ne serait alors pas simplement celui d'un être humain, il s'agirait en outre d'un kinan libéré à la suite d'une mort violente, et par conséquent doté de tous les attributs du kinan d'une victime d'homicide. C'est donc en toute logique que l'agression d'une puissance de jabun ne diffère pas en nature de celle du kinan d'un tué.

Néanmoins, le kinan introduit dans une puissance de jabun a la particularité de faire l'objet de manipulations rituelles, de sorte que, contrairement à celui que doit affronter un meurtrier, il est soumis à un certain contrôle. Ces manipulations rituelles consistent pour partie à raviver cette puissance de kinan par l'immolation d'un animal, et à faire participer à son action des principes extraits de certains végétaux. Tous ces éléments animaux, végétaux et humains contribuent à la constitution de la puissance de jabun. C'est néanmoins le kinan d'un homme (en outre probablement décédé de mort violente) qui en constitue la pièce maîtresse, car c'est à ce kinan que jabun doit de pouvoir s'affronter à un autre kinan, celui de la victime du meurtre.

41 Mais s'agit-il réellement de deux kinan distincts ? Le jabun incorporé par le meurtrier ne participe-t-il pas plus intimement du kinan de la victime ? On pourrait en effet formuler l'hypothèse que la cure ne serait autre chose que la maîtrise, par des moyens rituels, d'une puissance émise par la victime, puissance qui se dédoublerait en deux entités : d'une part un kinan hostile à maintenir à distance et d'autre part une puissance incorporée par le meurtrier, versant en quelque sorte domestiqué du kinan de la victime. L'utilisation d'un os et d'une portion de foie provenant d'un homme tué par flèche ne serait alors pas seulement motivée par la nécessaire introduction, au cœur de la puissance de jabun, de la force du kinan humain d'une victime anonyme de meurtre ; elle serait une manière de faire participer à la cure du meurtrier sa propre victime. Mais comment, dans cette hypothèse, expliquer le cas d'un homme qui, ayant fait l'acquisition d'une puissance de jabun sans avoir commis de meurtre, se voit attaqué par cette puissance ? D'où cette dernière tire-t-elle ce pouvoir de tuer l'imprudent si ce n'est dans un kinan qui ne peut être qu'étranger à celui de sa victime, puisque, précisément, celle-ci fait défaut dans le cas présent?

Afin de mieux cerner ce rapport entre le kinan de la victime et celui qui est à la base du jabun que sollicite le meurtrier, il peut être utile d'engager une comparaison entre le rite de jabun et ceux mis en œuvre dans des contextes proches, mais différents. C'est d'ailleurs ce que suggèrent les Bassar eux-mêmes lorsqu'ils soulignent le lien qui existe entre le domaine du meurtre et celui des jumeaux. Les dernières séquences du rite de jabun nous serviront de fil conducteur. Elles mettent en évidence l'importance de cette proximité que le repas rituel établit entre le meurtrier et la puissance en vue d'écarter le kinan de la victime. Une fois le repas consommé (il ne doit pas l'être entièrement), le bénéficiaire du rite se saisit de la calebasse de jabun, qui contient les reliefs du repas 
rituel, et ramasse les plumes, les épluchures d'igname et les déchets des différents ingrédients qui ont servi à la préparation de la sauce. L'ensemble doit être abandonné au carrefour. On dit que c'est la part destinée à la victime. Mais cette opération ne saurait être réalisée sans précautions. C'est pourquoi, après s'être emparé de la corne et $\mathrm{du}$ couteau rituels, le jabundaan ouvre la marche. Lorsqu'il franchit le seuil de son vestibule, il marque une pause pour tracer, avec une crème composée de la poudre de jabun mélangée à du beurre de karité, une croix sur le sol. C'est là la seconde utilisation de la poudre dans le cadre de la cure, après son usage en tant que médicament destiné à être absorbé (cf. supra). Ensuite, tout au long du trajet, il peut tracer autant de croix qu'il juge nécessaire. Lorsqu'ils arrivent au carrefour, le jabundaan trace une dernière croix sur le sol, y plante le couteau, dépose la corne à proximité et demande au tueur de poser son pied gauche par-dessus, tout en renversant la calebasse pour la vider de son contenu. Les deux protagonistes redressent ensuite la calebasse ainsi vidée, puis chacun rentre chez soi. La part qui vient d'être abandonnée est destinée au kinan de la victime. En la consommant, celui-ci se rend impuissant, il perd tout pouvoir d'agir contre le meurtrier.

Ce double geste (renverser / redresser un récipient) figure fréquemment dans les rites bassar. Comme dans le cas présent, il apparaît dans les derniers moments d'une séquence rituelle comprenant une offrande: après la remise de celle-ci à l'instance sollicitée, après la consommation, par le bénéficiaire, de la part qui lui revient, arrive ce moment où est versée sur le sol une part qualifiée de "mauvaise» (tikpitil) sur laquelle on "ferme» (ki cikin) avant d'« ouvrir» (ki cikiti) sur le "bien" (tijan). Cette opposition, sur laquelle les formules et les gestes insistent de manière redondante, révèle bien la finalité de l'opération : en rejetant le mal, on laisse une place vacante que vient occuper le bien. Dans le contexte qui nous intéresse (celui de jabun), la position spécifique de cette opération est doublement soulignée : d'abord par la division spatiale qui l'isole du corps principal du rite, tout entier cantonné dans la cour du jabundaan, ensuite par la désignation explicite de l'instance destinataire, le kinan de la victime, à cette occasion dénommée "le mauvais ». Dans la plupart des autres cas, en effet, la destination des restes (il s'agit en général de la lie de la bière de mil qui a servi aux libations) n'est pas aussi univoque ; surtout, elle ne fait pas intervenir de manière aussi nette une instance distincte de la puissance sollicitée. En outre, les croix tracées tout au long du trajet (qui, dit-on, servent à "barrer le chemin » au kinan de la victime) renforcent encore cette division spatiale, de sorte que l'objectif du rite est parfaitement clair: pour s'opposer aux tentatives d'intromission de la victime dans le corps du meurtrier, tentatives qui mettent à profit toutes les occasions où l'homicide s'alimente, il faut opérer une étroite conjonction, qui s'établit surtout sur le mode alimentaire, entre le meurtrier et la puissance et, immédiatement après, à l'aide des dernières potentialités qu'offre le même dispositif rituel (la destination à réserver aux restes du repas rituel), instaurer une nette séparation vis-à-vis de l'entité menaçante. On retrouve en somme, mais amplifié, le double mouvement déjà mis en œuvre par l'utilisation des noms propres respectifs de la victime et de la puissance: il faut rapprocher le meurtrier de la seconde afin de l'éloigner de la première.

Dans le domaine des rites de jumeaux, si proche, selon les Bassar, de celui de jabun, on retrouve ces articulations générales, avec cependant certaines différences qui résultent pour une part de légères variations significatives sur le même fond et, pour une autre part, de la combinaison avec une autre opération rituelle qu'il nous faudra expliciter. Pour mieux y parvenir, nous examinerons un dernier domaine rituel, celui de 
l'initiation à la divination, où cette seconde procédure est cette fois seule présente. Cette démarche nous permettra en outre de déceler les raisons de la présence de cette seconde procédure rituelle dans le cas des jumeaux et de son absence dans celui du meurtrier.

\section{Jumeaux et divination}

Dans les représentations que les Bassar se font de la procréation, la principale composante de la personne, le kinan, est attribuée à l'enfant dès sa conception. Cette attribution est le fait d'une instance particulière qui, profitant des rapports sexuels qu'ont les parents, pénètre dans le corps de la femme et participe à la formation de l'enfant. La nature de cette instance dépend du comportement des parents dans les heures qui ont précédé leur accouplement. Dans la plupart des cas, ceux-ci ont passé la soirée dans la cour familiale et se sont couchés de bonne heure; c'est en tout cas la conduite socialement valorisée. Chez les Bassar, les ancêtres demeurent auprès des vivants, on dit qu'ils partagent les habitations de leurs descendants. C'est donc auprès d'eux que se sont trouvés les parents avant de s'unir, et c'est par conséquent l'un de ces ancêtres qui s'introduit dans le corps de la future mère. L'enfant aura alors un usindaan, un ancêtre tutélaire dont le nom sera identifié à sa naissance lors d'une consultation divinatoire spécifique, au cours de laquelle sera également révélé le choix prénatal du nouveau-né. On dit que c'est cet ancêtre qui a "sorti » l'enfant; c'est à lui que ce dernier est redevable de son kinan.

Mais il arrive que l'un des parents au moins ait fait preuve de négligence. Si par exemple, dans la journée qui précède, la femme a eu l'imprudence de manger soit à un carrefour, soit en marchant sur un chemin, surtout si ses mets sont des beignets préparés avec de l'huile rouge, elle a toute chance d'avoir attiré quelque esprit de brousse, un ukpalib, particulièrement friand de ce genre de nourriture. La nuit venue, si la femme a des rapports sexuels, cet ukpalib, resté à ses côtés, s'introduira alors dans son corps, se substituant ainsi à l'ancêtre qui aurait pu donner son kinan à l'enfant conçu. Dans d'autres cas, c'est le père qui commet la faute: elle consiste pour lui à circuler de nuit hors de l'espace habité avant de se coucher, à une heure particulièrement tardive, auprès de sa compagne. Lui aussi aura alors été suivi par un ukpalib $^{15}$. Dans les deux cas, l'enfant ainsi conçu, qui sera dépourvu d'ancêtre tutélaire, sera dit sambubiki, « enfant de malheur » (kibiki, " enfant », sambu, « malheur »). Parfois on en comptera deux, ce seront des jumeaux. Les autres enfants de la même catégorie se distingueront par un trait particulier, de ceux qui sont partout reconnus comme singuliers (enfants nés par le siège, nés coiffés, dotés d'un doigt ou d'un orteil en surnombre, conçus sans que la mère ait eu son retour de couches après la naissance antérieure, etc.). Ces signes révèlent que ce n'est pas un ancêtre, mais un être de brousse, qui a été à l'origine de la conception de l'enfant, lui donnant son kinan : on dira alors que c'est un ukpalib qui a « sorti » $l^{\prime}$ enfant $^{16}$.

47 Pour de tels enfants, on ne procède pas à la consultation divinatoire chargée d'identifier l'ancêtre tutélaire puisqu'une telle identification n'a pas lieu d'être. En lieu et place, on effectue une séance de divination très particulière dont se charge un spécialiste rituel ${ }^{17}$ et au cours de laquelle l'ukpalib donateur du kinan fait connaître son identité, mais aussi et surtout ses exigences quant aux rites qui devront être accomplis. Ces rites sont ceux dits de kuyuu, la calebasse. 
48 $k J)$. Son rôle est, principalement, de donner satisfaction à l'ukpalib qui s'est introduit dans l'espace des hommes au moyen de la conception puis de la naissance de l'enfant. Donner satisfaction à cet esprit de brousse, c'est l'«arranger ", ki yajki. L'enjeu porte notamment sur la santé de l'enfant. Il intéresse également la survie de ses géniteurs dans la mesure où l'ukpalib qui n'aurait pas été satisfait de la cérémonie destinée à $l^{\prime}$ « arranger » risque fort de s'en prendre à leur vie. Mais la venue au monde d'un sambubiki menace aussi ses géniteurs dans un autre registre. Ceux-ci ont avant tout à craindre de ne pouvoir subvenir aux besoins matériels de leur famille : dans le sillage de l'ukpalib qui a su ainsi s'introduire auprès des hommes, se pressent en effet plusieurs de ses «amis » qu'il invite à dévorer les produits du champ de son "père " humain. Quelle que soit l'ardeur que ce dernier mettra à cultiver sa parcelle, il ne pourra en accroître la production et aura toutes les peines du monde à assurer la subsistance des siens. Et il en va de même de toutes les activités productrices qu'il peut entreprendre, ainsi que de toutes celles dans lesquelles sa femme est engagée : si, par exemple, elle fait du commerce, elle sera incapable de réaliser le moindre bénéfice.

Ce sont donc deux ensembles de menaces qui pèsent sur la famille : d'un côté celles qui proviennent directement de l'ukpalib principal (sur la santé de l'enfant et la vie de ses géniteurs), de l'autre celles dont sont responsables les ikpalib secondaires qui l'accompagnent (sur la production des parents). Pour contrecarrer les unes et les autres, le rite de kuyuu met en œuvre deux procédures qui paraissent confondues en une seule tant elles sont intriquées, mais dont les finalités sont néanmoins discernables. Je ne décrirai pas ici l'ensemble du rituel ${ }^{18}$, me contentant de signaler les éléments les plus significatifs pour mon propos, qui portent tous sur la phase terminale. Comme pour jabun, la préparation d'une médecine est étroitement coordonnée à la série des offrandes remises à la puissance. Mais cette fois le rite se déroule chez l'intéressé et non chez le spécialiste sollicité (kuyuudaan), qui est obligé de se déplacer. À cette différence s'en ajoute une autre qui nous retiendra plus particulièrement. Au terme de la cérémonie, on asperge d'eau le sol pour qu'y restent collés les déchets produits: plumes des volatiles immolés, épluchures de racines, pelures d'ignames, etc. doivent être laissés toute la nuit dans la cour pour, disent les Bassar, témoigner de ce que la cérémonie $\mathrm{d}^{\prime}$ «arrangement» (dijaykil) a bien été accomplie. Parallèlement, la calebasse rituelle, kuyuu, est déposée à la tête du sambubiki (le cas échéant, à la tête des jumeaux) pendant toute la durée de son sommeil nocturne. Comme cette calebasse est le récipient dans lequel ont été servis les plats rituels préparés, et comme, de même que pour jabun, il est impératif que des reliefs de ces plats soient conservés pour la phase finale de la cérémonie (qui, dans le cas de kuyuu, ne se tient que le lendemain), ce sont aussi ces restes qui passent la nuit au chevet du bénéficiaire. Mais, ainsi que le fera apparaître la comparaison avec le dernier rite qui nous retiendra, celui relatif à l'initiation au pouvoir de la divination, ce trait paraît en fait accessoire : ce qui importe, c'est que la calebasse, en tant qu'élément rituel, reste toute la nuit auprès de l'intéressé. Son contenu ne semble jouer à ce moment aucun rôle précis. On mesure toute la différence avec la cérémonie de jabun, où il serait particulièrement irresponsable de laisser longtemps, de nuit de surcroît (période la plus dangereuse, on l'a vu), les restes aux côtés du bénéficiaire, en l'occurrence le meurtrier : ces restes étant destinés à sa victime, rien n'est plus urgent que de s'en débarrasser, ce qu'on fait en outre avec les précautions rituelles de rigueur. 
50 C'est donc le lendemain, dans la matinée, que les reliefs du repas médicinal de kuyuu deviennent un élément pleinement actif du rite. Dans cette ultime phase, les opérations accomplies se rapprochent à nouveau de celles observées dans le contexte de jabun. On commence par balayer la cour, entassant pêle-mêle les ordures ménagères et les déchets de la cérémonie de la veille. Le tout est joint aux restes du repas toujours contenus dans la calebasse rituelle. Le père de l'enfant sambubiki emporte ce récipient jusqu'à son champ d'igname ${ }^{19}$. À l'aide d'une crème composée de la poudre médicinale préparée lors du rite de kuyuu mélangée à une certaine quantité de beurre de karité, il trace quatre croix aux quatre extrémités de sa parcelle avant d'enterrer en son centre le contenu de la calebasse. Ces restes de pâte enfouis sont destinés aux « mauvais » que sont les ikpalib associés à l'enfant sambubiki, ceux-là mêmes qui sont responsables des ponctions considérables subies par le champ du père. En consommant cette part, ils perdent tout pouvoir d'effectuer le moindre prélèvement sur la production du champ.

51 Entre cette phase finale et celle où, au terme de la cure de jabun, le meurtrier et le jabundaan abandonnent au carrefour les restes de la cérémonie, les similitudes sont nombreuses. Il s'agit ici comme là d'opérer une séparation vigoureuse entre le bénéficiaire et une entité hostile, jugée dangereuse : le kinan de la victime dans le cas de jabun, les ikpalib dévastateurs dans le cas de kuyuu.

52 En revanche, les opérations qui, dans le rite de kuyuu, sont accomplies la veille à la tombée de la nuit se réfèrent à une procédure distincte, celle d'« arrangement ». C'est cette fois l'ukpalib principal qui est visé, l'objectif étant de satisfaire ses exigences, afin de l'apaiser. Cette dernière procédure, difficilement discernable dans le contexte de kuyuu en raison de son étroite imbrication avec celle d'expulsion, apparait de façon autonome dans le troisième complexe rituel relevant de l'ensemble des médecines détenues à titre individuel, l'initiation à la divination.

Les Bassar reconnaissent deux phénomènes à l'origine de l'acquisition du statut de devin. Soit l'individu a exprimé, au cours de sa vie prénatale, le désir d'exercer la fonction de devin; le contenu de ce choix prénatal (biwunlimbu) est alors révélé dès la consultation divinatoire faite peu après la naissance de l'enfant. Soit l'intéressé fait une rencontre accidentelle avec un esprit diwaal et cet événement lui impose son initiation à la divination. Dans les deux cas, il faut d'abord procéder à l'installation d'un pot rituel, kubJocuu, première étape d'un long processus qui conduira le futur devin aux différentes étapes de son savoir. Ce premier rite est indispensable pour assurer la bonne santé de l'enfant qui, jusque-là, est constamment « dérangé ». Il s'agit donc, là encore, d'un rite d'« arrangement ». Mais cette fois, aucune procédure de séparation ou d'évacuation ne lui est associée. On se trouve donc, à première vue tout au moins, dans une situation inverse de celle en vigueur dans le domaine du meurtre. C'est ce que permet de confirmer l'observation des procédures rituelles correspondantes.

54 Comme dans les exemples précédents, le rite de kuboocuu consiste pour l'essentiel à effectuer un certain nombre d'offrandes sur le pot rituel du même nom et à y intégrer la préparation d'une médecine - dans ce cas réduite au jus d'une seule racine que l'on introduit dans la sauce du repas rituel. Les éléments distinctifs les plus significatifs concernent à nouveau la séquence terminale. Tout comme dans le cas de kuyuu, la cour est aspergée d'eau au terme de la cérémonie afin que les déchets y restent toute la nuit ; simultanément, le pot kuboวcuu est déposé au chevet de l'enfant où il est laissé également jusqu'au matin. Ici encore, les Bassar disent qu'il s'agit de témoigner de ce que la cérémonie qui «dérangeait » l'enfant a été dûment "arrangée ». Le lendemain 
matin, les déchets sont rassemblés et jetés au carrefour. Mais cette ultime opération, capitale dans les contextes de kuyuu et de jabun, est cette fois réduite à sa plus simple expression : en particulier, il n'est fait aucun usage d'une poudre médicinale - qui n'est d'ailleurs pas préparée - pour ponctuer un parcours ou délimiter un espace.

Les opérations qui composent la phase finale du rite de kuboocuu reprennent par conséquent une partie de celles propres au rite de kuyuu. Il se trouve qu'elles sont en outre précisément celles qui, dans le domaine de kuyuu, n'avaient pas leurs correspondantes dans celui de jabun. Inversement, d'un point de vue formel, kuboocuu se distingue de kuyuu principalement par l'absence de ces opérations communes à jabun et à kuyuu. De sorte que kuyuu se révèle comme un rite qui combine deux procédures qui ailleurs sont dissociées et auxquelles les rites de jabun et de kubJocuu, respectivement, se sont cantonnés.

On a donc deux procédures discernables qui interviennent soit distinctement (cas de jabun pour l'une, de kuboocuu pour l'autre), soit simultanément et de manière articulée (cas de kuyuu). La première procédure opère à partir des reliefs de repas et de la préparation d'une poudre médicamenteuse, d'abord destinée à être absorbée, puis ultérieurement utilisée sous forme de crème pour tracer sur le sol des croix dont le rôle est de «barrer le chemin » aux " esprits mauvais »; cette première procédure s'achève enfin par l'expulsion de ces restes de repas hors du domaine habité afin d'éloigner des entités menaçantes rendues simultanément moins agressives. L'autre procédure, dont la manifestation rituelle s'observe essentiellement dans l'obligation de laisser toute la nuit les déchets de la cérémonie sur le sol de la cour et dans celle de déposer dans le même temps au chevet de l'enfant les ustensiles rituels fondamentaux du rite (le pot kubojcuu dans un cas, la calebasse rituelle kuyuu dans l'autre), s'attache à "arranger " une entité qui « dérange ». Dans ce canevas global, chacun des trois rites considérés occupe une place spécifique: jabun en optant exclusivement pour la première procédure, kubojcuu pour la seconde et enfin kuyuu pour une combinaison des deux.

Un dernier critère doit être examiné, celui du lieu où se déroule la cérémonie. Dans le cas de jabun, on l'a vu, le meurtrier doit se rendre chez le détenteur de la puissance. $C^{\prime}$ 'est en effet chez ce dernier que se trouve le foyer rituel $\mathrm{gfan}$, élément fixé dans le sol de sa cour et qui interdit par conséquent toute mobilité. En revanche, dans le cas de kub oscuu, le rite a lieu chez l'intéressé, et c'est le ritualiste qui se déplace. Le rite inaugural du cycle correspondant est à cet égard désigné de manière très significative par l'expression "installer le pot kuboocuu» (ki yoon kubojcuu), sous-entendu "chez le bénéficiaire ». Il s'agit donc d'opérer une inscription dans un espace particulier, et le choix de cet espace n'est pas indifférent. Ces deux localisations distinctes renvoient bien évidemment au fait que les finalités des deux rites sont opposées : dans un cas tout est mis en œuvre pour éviter le moindre contact entre le meurtrier et sa victime, tandis que dans l'autre on s'accommode de la présence du diwaal dont il faut se ménager les faveurs et qui, de toute façon, est appelé à séjourner désormais auprès de son détenteur.

Dans le cas de kuyuu, comment est résolue la question du lieu où doit se dérouler la cérémonie? On observe que l'option retenue s'aligne sur celle de kuboวcuu: le rite a lieu non chez le spécialiste, mais dans la famille du sambubiki. En outre, le rite inaugural est dit "fixer la calebasse" (ki baan kuyuu), expression qui, comme dans l'exemple précédent («installer kuboocuu»), souligne bien l'implantation de l'élément central du rite dans la demeure du bénéficiaire. C'est que, des deux procédures conjointement à 
l'œuvre dans le domaine de kuyuu, celle d'arrangement l'emporte sur celle de séparation : la coexistence avec l'enfant sambubiki et l'ukpalib qui lui a donné son kinan est inévitable et il faut, là encore, trouver un accommodement, faute de quoi la vie de certaines personnes s'en trouverait menacée; en revanche, l'éloignement des ikpalib secondaires, compagnons de l'ukpalib principal, pour nécessaire qu'il soit, n'est pas aussi crucial que l'est celui de la victime dans le contexte du meurtre, car la menace qu'ils font peser ne concerne pas la vie des habitants de la cour, mais seulement la production de leur champ.

i on considère la place occupée par le complexe rituel du meurtrier dans l'ensemble des rites qui relèvent de la même catégorie, on observe donc qu'il est tout entier concentré dans une procédure d'éloignement et de séparation vis-à-vis d'une entité hostile alors qu'il se situe dans un contexte où une telle procédure est pensée comme l'un des termes d'une alternative dont l'autre terme serait un accommodement (un " arrangement ", disent les Bassar) vis-à-vis d'une entité qui "dérange " mais avec laquelle la cohabitation est incontournable. Ainsi, d'un point de vue formel tout au moins, l'option non réalisée selon laquelle l'esprit de la victime d'un meurtre serait intégrée et "arrangée », dotant l'homicide de pouvoirs nouveaux, n'est pas intrinsèquement impensable dans le système de pensée bassar. Tout semble en place, au contraire, pour qu'une telle transformation soit envisageable. On se serait ainsi trouvé dans une situation à certains égards proche de celle familière aux sociétés amazoniennes où le meurtre est un moyen de se procurer chez la victime les substances ou les principes nécessaires à l'acquisition de pouvoirs spécifiques. Toutefois, la distance est grande qui sépare le pensable du possible, et il va sans dire qu'une telle transformation, pour devenir effective, supposerait en outre un bouleversement profond de pans entiers de l'organisation sociale et symbolique des Bassar.

En déclarant que l'emprunt du dispositif rituel de kuyuu auprès de leurs voisins kotokoli les amena à modifier doublement le traitement qu'ils réservaient jusque-là aux jumeaux, les Bassar ne faisaient qu'exprimer à leur manière les relations complexes qui lient les deux domaines rituels. Rappelons en effet que, selon leur commentaire en forme de petit récit, ce changement avait consisté à la fois en un transfert du problème à traiter depuis le domaine de jabun vers celui de kuyuu et en l'abandon de la pratique de mise à mort des jumeaux au profit d'une intégration de ces derniers à l'unité familiale. En d'autres termes, une transformation sur le plan des pratiques rituelles s'accompagnait d'une modification identique quant au sort à réserver aux intéressés. Kuyuu réalisant sur le plan rituel une intégration là où jabun n'opérait qu'une expulsion, il devenait simultanément possible de conserver en vie des jumeaux qui jusque-là étaient rejetés en brousse. Le petit récit rapporté par les commentateurs bassar avait certes pour objet de mettre en exergue le lien qui associe les deux dispositifs rituels, mais il visait dans le même temps à souligner leurs différences : c'est en tout cas ce que suggère la mention d'un changement de comportement à l'égard des jumeaux. Jabun et kuyuu ont bien pour vocation commune de traiter des rapports que quelques personnes singulières entretiennent avec certaines entités déterminées (kinan d'une victime dans le premier cas, esprit de brousse ukpalib dans le second), mais tandis que l'une le fait au moyen d'une séparation, l'autre le fait par le truchement d'une intégration, possible dès lors que l'« arrangement » requis a été accompli. Et, au-delà de ces procédures rituelles, ce sont des attitudes générales tout aussi contrastées qui sont dépendantes du choix effectué. 
61 Pour s'en tenir au cas du traitement du meurtrier, le choix de l'expulsion est d'autant plus significatif qu'il semble résumer bon nombre des spécificités bassar de la question. Comme dans de nombreuses sociétés, le meurtre déclenche d'abord une soudaine proximité entre le meurtrier et sa victime, proximité qui tend vers la fusion, entraînant une confusion de leur identité respective ; en témoignent notamment les descriptions faisant état des images de sa victime qui hantent de façon obsédante le meurtrier. Mais alors que ces phénomènes passent généralement pour constituer le cours normal du changement d'état du tueur, l'objet de la cure consistant simplement à accompagner cette transformation au moyen d'un appareil rituel minimal, ils sont pour les Bassar le signe que quelque chose de terrifiant est en train de se produire. Et tout l'objet de la cure, encadrée par un imposant dispositif rituel, est chez eux de stopper net cette évolution. S'ils viennent à se manifester, de tels symptômes ne se résorbent pas d'euxmêmes ; tant que rien n'est fait pour les contrecarrer, ils s'amplifient et vont jusqu'à la mort du meurtrier. Cette mort n'est pas, comme dans certaines sociétés, une mort symbolique suivie d'une renaissance, elle est irréversible et même caractérisée comme mauvaise mort. Afin d'éviter une telle issue, le seul moyen consiste à interdire tout contact entre le meurtrier et l'esprit de la victime, puis d'expulser ce dernier : c'est précisément ce qui est attendu du rite de jabun.

On est donc loin, chez les Bassar, d'un processus d'incorporation de l'esprit de la victime qui, au terme de transformations successives, deviendrait le double du meurtrier, dont il modifierait partiellement l'identité. Cela ne veut pourtant pas dire qu'aucune altération n'affecte l'homicide bassar. Nous avons vu en effet que la mise à distance de l'esprit hostile de la victime n'était possible qu'au prix de l'ingestion d'une puissance de même nature, puisque constituée autour du kinan d'un être humain, probablement lui aussi victime d'homicide. Il faut donc, malgré tout, qu'une fusion s'opère avec le kinan d'une victime, mais sous deux conditions restrictives: d'abord cette victime doit être distincte de celle du meurtrier, ensuite cet autre kinan ne peut être incorporé que par le biais de la puissance de jabun qui en assure la maîtrise rituelle. L'esprit de la victime n'est cependant pas totalement étranger à la mutation que subit l'homicide puisqu'il interagit avec le kinan que ce dernier a incorporé : ce kinan ne peut être durablement supporté que s'il est confronté à son antagoniste, qui est précisément celui de la victime.

On se trouve en définitive devant une configuration à trois termes qui se substitue à la paire classique associant le tueur et l'esprit de sa victime : cette paire est ici augmentée du troisième terme que constitue la puissance de jabun, formée autour du kinan d'une tierce personne. Entre ces trois termes, deux relations duelles s'observent: l'une associant le kinan de la victime et la puissance de jabun, qui sont deux entités de même nature entre lesquelles un équilibre doit être trouvé, l'autre liant le meurtrier à la puissance de jabun, désormais intégrée à sa personne. L'unique relation qui n'est pas réalisée, parce qu'elle est précisément l'objet de tous les évitements, est celle qui, ailleurs, est habituellement la seule attestée : l'association complexe entre le tueur et l'esprit de sa victime.

Cette configuration à trois termes montre que si jabun est un rite qui, notamment par rapport aux rites apparentés de kuyuu et kuboวcuu, gravite pour l'essentiel autour d'une puissante procédure de séparation, il ne s'y réduit pas : il comporte en outre une procédure d'incorporation, qui fait intervenir un troisième élément. Cette articulation est du reste bien mise en évidence par certaines séquences du rite, comme le jeu autour 
des noms propres de la puissance et de la victime, ou encore les traitements contrastés des parts alimentaires destinées respectivement au meurtrier et à sa victime : à chaque fois, le rapprochement entre la puissance et le tueur se combine avec un éloignement entre ce dernier et l'esprit de sa victime. Par conséquent, même si jabun se singularise avant tout par la mise en œuvre d'une procédure de séparation, il partage avec les deux complexes rituels qui lui sont apparentés une mutation du bénéficiaire (respectivement meurtrier, sambubiki, devin) sous l'effet d'une relation quasi-fusionnelle avec une entité spirituelle (respectivement kinan de la puissance de jabun, être de brousse ukpalib, esprit diwaal). Mais, alors que cette entité - ce double - est donnée d'elle-même dans les deux derniers cas, son intégration se limitant alors à un " arrangement ", elle est, dans celui de jabun, construite pour l'essentiel dans le cadre de la cure. Elle n'est cependant pas un pur produit de l'édification rituelle puisqu'elle se définit également dans sa confrontation avec l'entité déjà-là du domaine de jabun, l'esprit de la victime. C'est par cet équilibre subtil entre une entité hostile qu'on ne peut se concilier et une entité assimilable mais que l'on se doit de construire que les Bassar ont résolu la redoutable question du devenir de leurs meurtriers.

\section{BIBLIOGRAPHIE}

Bonnet, Doris (1982) « La procréation, la femme et le génie (les Mossi de Haute-Volta) », Cahiers ORSTOM, série Sciences Humaines XVIII (4) : 423-431

Bonnet, Doris (1988) Corps biologique, corps social. Procréation et maladies de l'enfant en pays mossi, Burkina Faso, Paris, ORSTOM .(« Mémoires » 110)

Bonnet, Doris (1994) « L'éternel retour ou le destin singulier de l'enfant », L'Homme XXXIV (3) : 93-110.

Dugast, Stéphan (1992) Rites et organisation sociale. L'agglomération de Bassar au Nord-Togo, thèse de doctorat, Paris, EHESS.

Froelich, Jean-Claude (1954) La tribu konkomba du Nord-Togo, Dakar, IFAN (« Mémoires » 37).

\section{NOTES}

1. Michel Cartry a eu l'amabilité de me faire part de ses réflexions sur les matériaux dont il est question ici, d'abord à partir des passages de ma thèse qui en traitent, ensuite à propos d'une première version de ce texte. Ses commentaires, toujours féconds, m'ont été d'une grande utilité dans l'élaboration de certaines propositions. Qu'il en soit ici vivement remercié.

2. Dans un article paru récemment sur les différentes catégories conceptuelles relatives à l'enfant en bas âge chez les Mossi (population relevant, au même titre que les Bassar, de l'aire culturelle dite voltaïque), D. Bonnet rapporte des pratiques similaires (exposition de certains types d'enfants à l'intérieur d'une termitière ou dans le creux d'un arbre). Cependant, elle fait observer que, dans la pensée mossi, «il ne s'agit pas, pour les personnes à l'origine du décès, d'un crime puisque l'enfant n'est pas considéré comme une personne» (Bonnet 1994 : 99-100). En effet, ce 
statut de personne à part entière ne lui sera reconnu qu'une fois qu'il aura franchi les étapes décisives que sont le sevrage et l'acquisition du langage. Les représentations des Bassar diffèrent sur ce point nettement de celles des Mossi : dès sa naissance, l'enfant bassar est pourvu de tous les attributs qui en font une personne, notamment de celui qui lui permet de se venger en cas de mort violente, le kinan. Et, par rapport aux Mossi, c'est bien d'une véritable mise à mort dont il est question chez les Bassar car l'enfant est chez eux enterré vivant et non simplement exposé.

En ce qui concerne les représentations de la personne, nous verrons que, plus largement, la conception que les Bassar se font de la formation d'un enfant se démarque sensiblement de celle élaborée par les Mossi, et ce en dépit de représentations élémentaires identiques; les différences recensées n'en sont que plus significatives.

3. Certains informateurs ajoutent que, de retour chez lui, afin de protéger les parents des jumeaux - ceux-ci ayant une part de responsabilité dans le meurtre, même s'ils n'en étaient pas directement les auteurs-, ce spécialiste leur faisait lécher la médecine de jabun. D'autres informateurs contestent vigoureusement une telle version.

4. Bien que les maîtres de la foudre ne prennent en charge qu'un seul cas, celui des foudroyés, leur champ de compétence s'articule lui aussi en deux catégories renvoyant à l'articulation "brûlés » / " enflés ». Les Bassar distinguent en effet deux types de foudre et, partant, deux types de foudroyés : aux victimes de «la foudre qui frappe » (utaatookaa), dont les corps sont calcinés, s'opposent celles de «la foudre qui remplit» (utaafunkaa), dont les cadavres présentent un gonflement général ( $c f$. Dugast 1992 : chap. VII, plus particulièrement pp. 608-616).

5. Il en va différemment de certaines puissances détenues collectivement par quelques communautés déterminées, $c f$. Dugast 1992 : chap. VII.

6. Comme de nombreuses autres sociétés d'Afrique de l'ouest, les Bassar associent le chiffre 3 à la masculinité et le chiffre 4 à la féminité.

7. Certains informateurs précisent qu'il ne peut s'agir que de l'os d'une personne tuée par flèche. Selon d'autres, cette particularité n'est pas exigée, n'importe quel os d'un bras gauche humain pouvant faire l'affaire.

8. Ces animaux forment une partie de ceux dont la mise à mort implique, pour le chasseur, l'accomplissement du rite de jabun, au même titre que s'il s'agissait d'un homicide (sur l'équivalence entre les deux types d'acte, voir Dugast 1992 : 387-391).

9. C'est le meurtrier lui-même qui doit se charger de puiser cette eau. Son entreprise n'est pas sans risque. Comme dans tous les moments critiques de la cure, le principal danger est que le kinan de la victime s'introduise dans les aliments ou les breuvages qu'absorbe le meurtrier. Dans le cas présent, il est impératif de tenir ce kinan éloigné de l'eau qu'il pourrait contaminer. A cette fin, le meurtrier attache des feuilles de néré autour du récipient qui lui servira à puiser l'eau. Pour plus de précautions, il emporte avec lui le couteau de jabun ou la corne rituelle appartenant au jabundaan auquel il s'est adressé.

10. Pour certaines puissances qui l'exigent, les plumes ou les poils de l'animal sont en outre arrachés et, dans ce cas, ils doivent être collés sur tous les objets déposés au sol.

11. Les informateurs sont divisés quant aux caractéristiques de cet organe. Leurs divergences sont vraisemblablement le reflet des variations entre les différentes puissances de jabun. Selon les uns, il est indispensable que le foie provienne d'un homme tué par flèche, selon les autres, cela n'est pas nécessaire.

12. C'est à nouveau un point sur lequel tous les informateurs ne sont pas unanimes, certains déclarant qu'aucune portion de foie n'est requise dans cette préparation.

13. Certains détenteurs de jabun affirment toutefois n'utiliser que la poudre médicinale dans ces cas-là.

14. Notamment le vautour, autre animal dont la mise à mort implique la mise en œuvre de la cure de jabun. 
15. Autrefois, lorsqu'un homme tardait à rentrer chez lui, un vieux ou une vieille de la cour allait chercher son épouse pour la faire dormir à ses côtés : ainsi, à son retour, son mari ne la trouvait pas. C'était là une manière de soustraire la femme à l'intrusion d'un ukpalib.

16. Sur ce point, la conception des Bassar se différencie très nettement de celle qu'ont élaborée les Mossi. Pour ces derniers, l'intervention d'un génie est indispensable à la procréation, la formation d'un enfant requérant la participation conjointe d'un ancêtre et d'un génie. Une telle complémentarité s'oppose à la concurrence que se livrent, chez les Bassar, ancêtres et génies autour de la procréation : tous deux se disputent l'octroi de la même composante de la personne, le kinan. Il est d'ailleurs significatif que le même acte qui, chez les Bassar est assimilé à une faute ayant pour conséquence la naissance d'un enfant hors normes, est au contraire recommandé chez les Mossi pour toute femme qui désire être enceinte : il lui suffit d'attirer un génie avec des aliments sucrés afin qu'il la suive jusqu'à sa concession où il attendra qu'elle ait un rapport sexuel pour s'introduire en elle et la féconder (Bonnet 1982: $426 ; 1988: 25$ ). Il résulte de ces conceptions que, si les Mossi considèrent eux aussi les jumeaux comme des enfants plus proches des génies que ne le sont les enfants ordinaires, l'écart est chez eux bien moindre que celui conçu par les Bassar puisque tout enfant mossi est associé à un génie.

17. Un devin qui a acquis en outre la compétence particulière requise pour ce type de consultation.

18. Présenté ailleurs (Dugast 1992 : 369-381).

19. Le champ d'igname est celui qui, chez les Bassar, ouvre un nouveau cycle de culture et qui, comme tel, est considéré comme particulièrement exposé aux diverses menaces d'ordre surnaturel.

\section{RÉSUMÉS}

Chez les Bassar (Togo), les affaires de meurtre relèvent de la même catégorie rituelle que celles des jumeaux et des devins. Cet ensemble s'organise autour de deux procédures essentielles: l'assimilation et l'expulsion. Le domaine du meurtre se distingue par un recours privilégié à la seconde de celles-ci. En effet, avant de bénéficier d'un quelconque prestige, le meurtrier est d'abord un individu exposé à une mort prochaine. L'accent est donc mis sur le traitement rituel qui lui permet d'échapper à ce sort, plutôt que sur les représentations élaborées autour de ce que serait son nouveau statut, après acquisition de pouvoirs supplémentaires. Cette formule n'est pourtant pas la seule envisageable : du fait de son insertion dans un contexte rituel plus large, la configuration bassar aurait pu présenter une toute autre physionomie au prix d'une simple transformation, substituant l'assimilation à l'expulsion.

Among the Bassar (Togo), murder cases fall in the same ritual category as those of twins and diviners. This set of rituals is organized around two basic ritual procedures: assimilation and expulsion. Murder primarily involves recourse to expulsion. Before obtaining any prestige from his action, the murderer is an individual exposed to a sudden death. Emphasis is laid on the ritual treatment for escaping this fate, rather than on the ideas surrounding the new status obtained after acquiring additional powers. This is not the only conceivable configuration: by placing the Bassar case in a broader ritual context, it could assume a totally different form were a simple transformation performed so as to replace expulsion with assimilation. 
INDEX

Population Bassar

Mots-clés : meurtre, jumeaux, devin

Keywords : murder, twins, diviners

Index géographique : Togo

\section{AUTEUR}

STÉPHAN DUGAST

O.R.S.T.O.M 